\title{
Study of ovarian reserve parameters in infertile women of different age groups
}

\author{
Umakant Valvekar ${ }^{1 *}$, Santhana Lakshmi ${ }^{1}$, Amar Nagesh Kumar ${ }^{2}$
}

\begin{abstract}
${ }^{1}$ Department of Obstetrics and Gynecology, ${ }^{2}$ Department of Biochemistry, Karpaga Vinayaga Institute of Medical Sciences and Research Center, Chinakolambakkam, Madhurantagam, Tamilnadu, India
\end{abstract}

Received: 04 June 2016

Accepted: 06 June 2016

\section{*Correspondence:}

Dr. Umakant Valvekar,

E-mail: urvalvekar@gmail.com

Copyright: (c) the author(s), publisher and licensee Medip Academy. This is an open-access article distributed under the terms of the Creative Commons Attribution Non-Commercial License, which permits unrestricted non-commercial use, distribution, and reproduction in any medium, provided the original work is properly cited.

\begin{abstract}
Background: Main causes of infertility include diminished ovarian reserve. Diminished ovarian reserve is about availability of number of good quality pre ovulatory oocytes in the ovaries. As a woman ages, her ovarian reserve decreases. In recent years assessment of "ovarian reserve" became a strategy in treatment of female infertility. Although the true and functional ovarian reserves reflect different stages of follicular development, they are inherently linked and both decline in parallel with increasing age. Accurate measurement of the ovarian reserve has long been a quest in reproductive medicine. The objective of the study was to find out the ovarian reserve and also to correlate the ovarian reserve indicators with different age groups of infertile women.

Methods: This prospective study was conducted at Karpaga Vinayaga Institute of Medical Sciences, Madhurantagam, Chennai, Tamilnadu, India during the period of January 2014 to April 2016. The study comprises of 100 newly diagnosed PCOS women and they were all divided into three age groups. Group I consists of infertile women who were under the age of 26 years or less than 26 years. Group II consists of infertile women of age range between 27 to 35 years. Group III consists of infertile women who are 36 years and greater than 36 years of age.

Results: In the present study, ovarian reserve markers had shown significant variation with age in infertile women. Of the markers considered, progesterone, anti-mullerian hormone (AMH) and antral follicle count (AFC) exhibited significant correlation with age. Luteinizing hormone (LH), follicle-stimulating hormone (FSH) with AFC did not show any significant association with age compared to AMH, progesterone and AFC.

Conclusions: Our study results also indicate that serum AMH measurement is better predictor for the number of early antral follicles than conventional hormone measurements. Using AMH measurement in combination with AFC may improve the assessment of ovarian reserve for evaluating the fertility potential and monitoring infertility treatment.
\end{abstract}

Keywords: Infertility, Ovarian reserve, Antiullerian hormone, Antral follicle count, Progesterone

\section{INTRODUCTION}

Female reproductive function presumes that the ovary contains a finite number of oocytes within primordial follicles and their depletion precedes and indicates the approach of menopause. ${ }^{1}$ This pool of primordial follicles is formed during fetal life from approximately 18 weeks gestation. One of the main causes of infertility is diminished ovarian reserve. In other words ovarian reserve is presence of number of good quality pre ovulatory oocytes in the ovaries. As a woman ages, her ovarian reserve decreases. In recent years assessment of "ovarian reserve" became a strategy in treatment of female infertility. Accurate measurement of the ovarian reserve has long been a quest in reproductive medicine. In recent years a dramatic increase in research has been seen. A large part is fuelled by measurement of anti mullerian hormone $(\mathrm{AMH})$ is more accurate than the other hormones. While inhibin B shows good prediction of oocyte. ${ }^{2,3}$ It has therefore not supplanted folliclestimulating hormone (FSH) as the most widely used marker of the ovarian reserve despite the latter hormone's 
well-recognized limitations. The general purpose of ovarian reserve testing is to assess the quality and quantity of remaining oocytes in an attempt to predict the reproductive potential. The availability of a test capable of providing reliable information regarding a woman's individual ovarian reserve within a certain age category would enable the clinician to provide an individually tailored treatment plan. The ideal screening test should be reproducible with low intercycle and intracycle variablity. It should also demonstrate high specificity to minimize the risk of false positive determination of decreased ovarian reserve in a woman with normal ovarian reserve. ${ }^{4}$

There are different tests to diagnose ovarian reserve. Traditionally, the age, follicle stimulating hormone (FSH), mid leuteal progesterone, estradiol (E2) levels and antral follicle count (AFC) by ultrasound at early follicular phase were used for evaluation of ovarian reserve. At present the available tests for ovarian reserve include

- Biochemical markers: FSH, Estradiol, AMH, Inhibin B.

- Ovarian ultrasound imaging: AFC, Ovarian volume.

Since age is only a rough estimate of ovarian reserve, many tests, such as FSH, lutenizing hormone (LH), inhibin, antral follicle count (AFC) and total ovarian volumes, have been develop to predict ovarian reserve more precisely. A combination of tests can provide more accurate information of ovarian reserve. Hence we planned the present study to find out the ovarian reserve by measuring anti mullerian hormone (AMH), mid luteal progesterone levels, $\mathrm{LH}$, follicle stimulating hormone FSH and AFC and also to find out correlation of the ovarian reserve indicators with different age groups of infertile women.

\section{METHODS}

This prospective study was conducted at Karpaga Vinayaga Institute of Medical Sciences, Madhurantagam, Chennai, Tamilnadu, India during the period of January 2014 to April 2016. The study comprises of 100 newly diagnosed PCOS women and they were all divided into three age groups. Group I consists of infertile women who were under the age of 26 years or less than 26 years. Group II consists of infertile women of age range between 27 to 35 years. Group III consists of infertile women who are 36 years and greater than 36 years of age. Patient history, health problem, other details were collected from each participant by using a pre-tested questionnaire and the data was noted. The study was approved by Karpaga Vinayaga Institute of Medical Sciences, Institutional Ethics Committee, Madhurantagam, Tamilnadu, India. Written and informed consent was obtained from all the participants in the study.
Standard anthropometric data like age, height, weight were measured and noted from each subject. The BMI was calculated as the weight in kilograms divided by the square of height in meters. Women with primary infertility with pure polycystic ovary syndrome will be recruited for the study. Women with Secondary infertility, pelvic inflammatory diseases, endometriosis, other pelvic pathology and those women who are already on treatment are excluded from the study. Patients with previous ovarian surgery, polycystic ovarian syndrome (PCOS) and premature ovarian failure (POF) were also excluded.

Diagnosis of PCOS was made on the basis of the rotterdam criteria. ${ }^{5}$ Two out of three of the following are required for diagnosis: oligo and/or anovulation (defined by the presence of oligomenorrhea or amenorrhea); clinical and/or biochemical signs of hyperandrogenism (defined by presence of hirsutism (Ferriman-Gallwey score $\geq 6$ ), acne or alopecia, and/or elevated androgen levels) and polycystic ovaries by gynecological ultrasound. Women with secondary infertility, pelvic inflammatory diseases, endometriosis, other pelvic pathology and those women who are already on treatment are excluded from the study. Patients with congenital adrenal hyperplasia, cushing's syndrome, androgensecreting tumors, known hypothyroidism on treatment and intake of any medication affecting endocrinal parameters were excluded from the study.

On 2-3 days of spontaneous menstrual cycle, all patients had a transvaginal scan by the same investigator using 4$10 \mathrm{MHZ}$ multi-frequency ultrasound probe to assess the number of antral follicles, measuring $2-10 \mathrm{~mm}$ in size and counted in each ovary. The sum of both counts was the antral follicle count. Levels of LH, FSH and AMH were determined on the same days whereas progesterone levels were measured on the 21 st day of the menstrual cycle.

\section{Laboratory investigations}

Fasting blood samples were collected in plain tubes. About $5 \mathrm{ml}$ of blood was collected from the antecubital vein. Blood samples were centrifuged at $3500 \mathrm{rpm}$ for 10 min to separate serum. Hormones like AMH, LH, FSH and progesterone hormone was measured by enzyme linked fluorescent immunoassay (ELFA) method using on the mini VIDAS instrument.

\section{Reference ranges}

1. Anti mullerian hormone (AMH)

- Optimal fertility: 4.0 to $6.8 \mathrm{ng} / \mathrm{ml}$

- Satisfactory fertility: 2.2 to $4.0 \mathrm{ng} / \mathrm{ml}$

- Low fertility: 0.3 to $2.2 \mathrm{ng} / \mathrm{ml}$

- Very low fertility: 0.0 to $0.3 \mathrm{ng} / \mathrm{ml}$

- High levels: $>6.8 \mathrm{ng} / \mathrm{ml}$

\section{Progesterone}

- Follicular phase: 0.2 to $1.4 \mathrm{ng} / \mathrm{ml}$ 
- Luteal phase: 4 to $25 \mathrm{ng} / \mathrm{ml}$

- Menopause: 0.1 to $1 \mathrm{ng} / \mathrm{ml}$

3. FSH (Folicular stimulating hormone): 3.0-13.0 $\mathrm{mIU} / \mathrm{ml}$

\section{LH (Luteinizing hormone): $0.5-10.5 \mathrm{mIU} / \mathrm{ml}$}

5. AFC (Antral follicle count): Total $>9$ follicles in both the ovaries.

SPSS 20.0 version was used for statistical analysis. All the results were tabulated as mean and standard deviation. Comparisons between groups were performed by ANOVA, further post hoc comparisons were done by Tukey HSD test. All the variables followed normal distribution. The $\mathrm{p}$-value of $<0.05$ was considered to be statistically significant. Pearson correlation was done for the comparison of parameters among infertile women of three age groups. The $\mathrm{p}$-value of $<0.05$ was considered to be statistically significant.

\section{RESULTS}

A total of 100 infertile women were enrolled for the study. All the study subjects were divided into three age groups. Group I includes women of age 26 or less than 26 years, group II contains women between the age of 27 to 35 years and group III contains women of age 36 and above 36 years.

Table 1 shows the mean and standard deviation of body mass index (BMI), AMH, AFC, LH, FSH and progesterone levels in infertile women of three different age groups. The mean serum AMH levels were elevated in group I and group II while there is a slight decrease in group III.

Table 1: Statical analysis by ANOVA and post HOC comparison by Tukey HSD test comparisons of parameters in three age groups.

\begin{tabular}{|lllllll|}
\hline Age groups & BMI & AMH & AFC & Progesterone & LH & FSH \\
\hline Group I $\leq 26$ years & $26.12 \pm 3.759^{\mathrm{a}}$ & $15.62 \pm 5.464^{\mathrm{a}}$ & $13.48 \pm 2.41^{\mathrm{a}}$ & $7.80 \pm 1.352^{\mathrm{a}}$ & $7.08 \pm 1.639^{\mathrm{a}}$ & $5.12 \pm 1.159^{\mathrm{a}}$ \\
\hline Group II 27-35 years & $27.08 \pm 5.306^{\mathrm{a}}$ & $19.98 \pm 5.428^{\mathrm{b}}$ & $14.29 \pm 2.89^{\mathrm{b}}$ & $6.20 \pm 1.946^{\mathrm{b}}$ & $7.50 \pm 2.625^{\mathrm{a}}$ & $5.57 \pm 1.497^{\mathrm{b}}$ \\
\hline Group III >36 years & $27.55 \pm 2.970^{\mathrm{a}}$ & $17.86 \pm 6.369^{\mathrm{a}}$ & $15.64 \pm 2.248^{\mathrm{c}}$ & $4.72 \pm 0.857^{\mathrm{c}}$ & $9.97 \pm 2.645^{\mathrm{b}}$ & $8.47 \pm 2.734^{\mathrm{c}}$ \\
\hline
\end{tabular}

Note: Values are expressed as means \pm S.D. in each group. Values not sharing a common superscript differ significantly at $p<0.05$ (Post Hoc: Tukey HSD test).

AFC, FSH levels showed consistent increase with the increase in age of infertile women, whereas mid-luteal progesterone levels showed consistent decrease with the increase in age of infertile women. Figure 1 shows the comparison of $\mathrm{AMH}, \mathrm{AFC}$ and progesterone levels in infertile women of different age groups. Figure 2, 3 and 4 shows the mean plots of $\mathrm{AMH}, \mathrm{AFC}$ and progesterone levels in three age groups of infertile women.

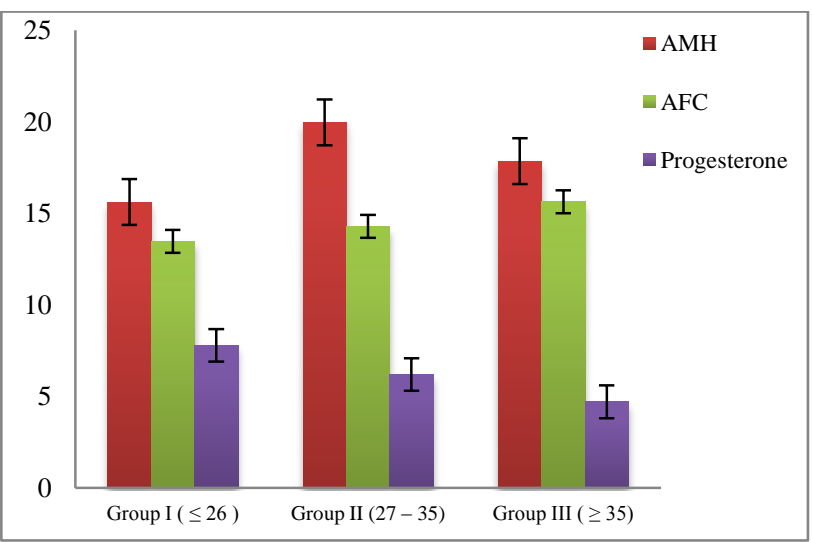

Figure 1: Comparison of AMH, AFH and progesterone levels among different age groups of infertile women.

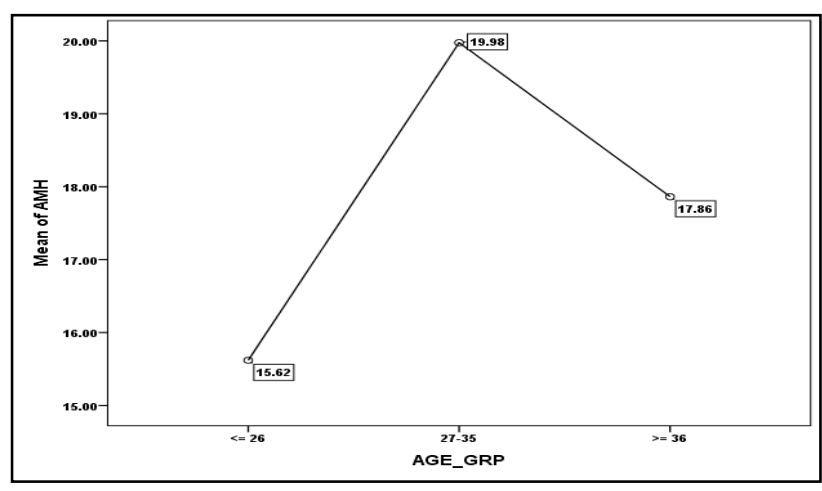

Figure 2: Mean values of AMH in three different age groups.

Pearson correlation study revealed that there is a strong negative correlation of progesterone with the age of infertile women $(r=-0.606$ and $\mathrm{p}<0.01)$ (Table 2). LH and FSH showed positive correlation with the age of infertile women $(r=0.295$ and $\mathrm{p}<0.01 ; \mathrm{r}=0.358$ and $\mathrm{p}<0.01$ respectively). AMH levels and antral follicle count showed strong positive correlation $(r=0.373$ and $\mathrm{p}<0.01)$ indicating that they are good markers for ovarian reserve in infertile women (Table 2). A negative correlation was observed between progesterone and FSH levels $(\mathrm{r}=-0.251$ and $\mathrm{p}<0.05)$. 


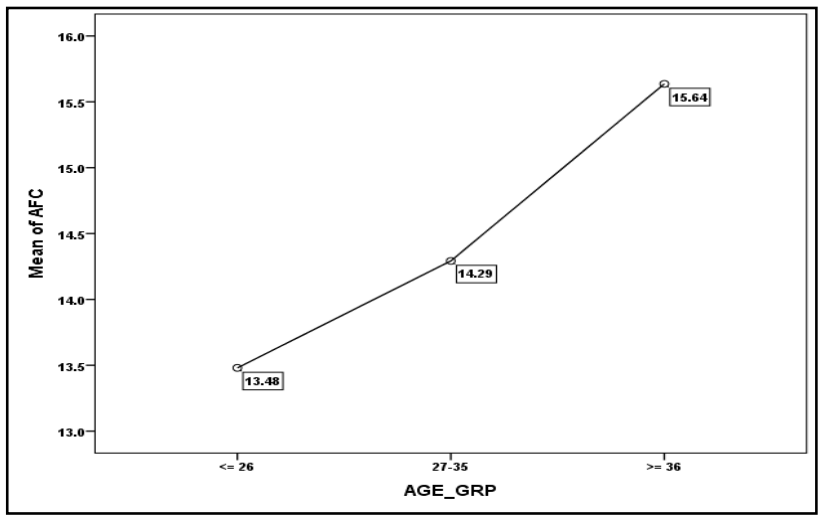

Figure 3: Mean values of AFC in three different age groups.

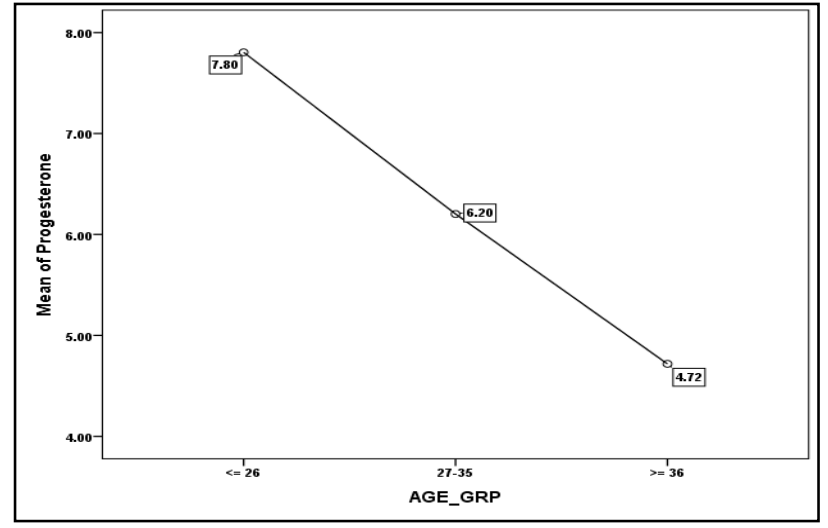

Figure 4: Mean values of progesterone in three different age groups.

Table 2: Pearson correlation test for all the parameters among infertile women.

\begin{tabular}{|llllllll|}
\hline \multirow{2}{*}{ Age } & Age & BMI & AMH & AFC & Progesterone & LH & FSH \\
\hline \multirow{2}{*}{ BMI } & 1 & 0.184 & 0.076 & $0.196^{*}$ & $-0.606^{* *}$ & $0.295^{* *}$ & $0.358^{* *}$ \\
\cline { 2 - 7 } & -- & 0.066 & 0.447 & 0.050 & 0.00 & 0.003 & 0.000 \\
\hline \multirow{2}{*}{ AMH } & 0.184 & 1 & 0.156 & 0.098 & 0.015 & 0.030 & -0.082 \\
\cline { 2 - 8 } & 0.066 & -- & 0.120 & 0.329 & 0.884 & 0.769 & 0.413 \\
\hline \multirow{2}{*}{ AFC } & 0.076 & 0.156 & 1 & $0.373^{* *}$ & -0.027 & -0.115 & -0.104 \\
\cline { 2 - 8 } & 0.447 & 0.120 & -- & 0.00 & 0.792 & 0.253 & 0.301 \\
\hline \multirow{2}{*}{ Progesterone } & $0.196^{*}$ & 0.098 & $0.373^{* *}$ & 1 & 0.071 & 0.028 & 0.103 \\
\hline \multirow{2}{*}{ LH } & 0.050 & 0.329 & 0.000 & -- & 0.480 & 0.783 & 0.307 \\
\hline \multirow{2}{*}{ FSH } & $-0.606^{* *}$ & 0.015 & -0.027 & 0.071 & 1 & -0.182 & $-0.251^{*}$ \\
\cline { 2 - 8 } & 0.00 & 0.884 & 0.792 & 0.480 & -- & 0.068 & 0.011 \\
\hline & $0.295^{* *}$ & 0.030 & -0.115 & 0.028 & -0.182 & 1 & 0.162 \\
\hline & 0.003 & 0.769 & 0.253 & 0.783 & 0.068 & -- & 0.105 \\
\hline
\end{tabular}

From the data present in Table 2, it is evident that AMH, AFC and mid-luteal progesterone levels were having very good association. There AMH, AFC and progesterone levels are the reliable markers in diagnosis and prognosis of infertility assessment in different age groups.

\section{DISCUSSION}

In the present study, ovarian reserve markers had shown variation with age in infertile women. Of the markers considered, progesterone, $\mathrm{AMH}$ and $\mathrm{AFC}$ exhibited significant correlation with age. LH, FSH with AFC did not show any significant association with age compared to $\mathrm{AMH}$, progesterone and AFC. Therefore, the data do not provide support for them as markers of ovarian reserve. The results obtained through our study show, that ovarian reserve assessment tests in each age group reflect age-specific changes. Above mentioned trends are also confirmed by other researchers. ${ }^{6,7,8}$ It is interesting to note, that in this study low serum AMH values was observed which was statistically significant in all the three age groups; whereas AFC values were statistically significantly higher in the group III compared with the group I, and in the group II compared with the group III; LH levels were statistically significantly higher only in group III compared with the group I. Thus, we can note that, AMH values better reflect age-specific changes, than other indicators. These findings were on par with the study done by de Vet et al. ${ }^{9}$ where in early follicular phase hormone measurements at 3 year intervals revealed that serum AMH levels decline significantly whereas, We examined relationships between the age and ovarian reserve indicators in the whole study group and found that: age is in high significant negative correlation with AMH level and AFC, and in high significant positive correlation with FSH. However, the relation between the age and FSH was moderate ( $\mathrm{rs}=0.38, \mathrm{p}<0.0001)$. Thus, with the age AMH and AFC values strongly decline and the FSH levels moderately increase. The results of de Vet 
et al. also suggest that changes in serum AMH levels occur relatively early in the sequence of events associated with ovarian aging. ${ }^{9}$ Elevated serum levels of FSH are not found until cycles become irregular. ${ }^{10}$ Therefore, a marker that already shows a considerable change when cyclicity is still normal, would better identify women with declining fertility. Above mentioned results strongly suggest, that serum AMH level can be used as a marker of ovarian aging.

In difference from the total study group comparison analysis within groups revealed quite interesting data in the group I and the most sensitive age group II (35-40 years), where the correlation between serum FSH levels and AFC was not statistically significant. Whereas AMH and AFC in all three study groups correlate positively and statistically significant. This positive correlation is confirmed by other researchers too. ${ }^{11,12}$

Despite the fact, that at present there is no agreement on identification of the antral follicles by size, the majority of researchers imply the amount of 2-10 mm follicles counted in early follicular phase. ${ }^{13,14}$ It has been reported that human antral follicles measuring $<6 \mathrm{~mm}$ express the great amount of $\mathrm{AMH}$, and levels decline with antral follicles increase in size. ${ }^{15}$ In the study by Goksedef et al, the best correlation was found between AMH levels and 5-6 mm antral follicles. ${ }^{16}$

In our study the number of 2-10 mm antral follicles was counted in early follicular phase and positive correlation between $\mathrm{AMH}$ and AFC values with high significance was found in all age groups. According to data of one of the recent studies there AMH has recently been proposed as a parameter to replace ultrasonographic assessment of PCO morphology, with specificity and sensitivity of 97.1 and $94.6 \%$ when using the rotterdam criteria, or 97.2 and $95.5 \%$ using the NIH criteria. ${ }^{17}$ Indeed, AMH levels correlate independently with both PCO morphology and androgenic profile. ${ }^{18}$ Another parameter proposed as an adjunct to PCO morphology is an assessment of the ovarian stromal volume, measured as a ratio of the stromal area to total area of the ovary (S/A ratio). Although this S/A ratio performed well when discriminating between women with and without PCOS, and correlated with androgen levels, it has not been adopted as part of any of the existing diagnostic criteria. $^{19,20}$

\section{CONCLUSION}

As our results indicate serum AMH levels are strongly related with AFC, among ovarian reserve assessment tests used in modern practice AMH levels should be considered to be more reliable. This study also reported that serum AMH measurement is better predictor for the number of early antral follicles than conventional hormone measurements. Using $\mathrm{AMH}$ measurement in combination with AFC may improve the assessment of ovarian reserve for evaluating the fertility potential and monitoring infertility treatment.

Funding: No funding sources

Conflict of interest: None declared

Ethical approval: The study was approved by the Institutional Ethics Committee

\section{REFERENCES}

1. Wallace WH, Kelsey TW. Human ovarian reserve from conception to the menopause. Public Lib Sci one. 2010;5:8772.

2. Yong PTK, Baird DT, Thong KJ. Prospective analysis of the relationships between the ovarian follicle cohort and basal FSH concentration, the inhibin response to exogenous FSH and ovarian follicle number at different stages of the normal menstrual cycle and after pituitary down-regulation. Hum Reprod. 2003;18:35-44.

3. Welt CK, Mcnicholl DJ, Taylor AE. Female reproductive aging is marked by decreased secretion of dimeric inhibin. J Clin Endocrinol Metab. 1999;84:105-12.

4. Testing and interpreting measures of ovarian reserve: a committee opinion. Practice committee of the american society for reproductive medicine. Fertil Steril. 2012;98:1407-15.

5. The rotterdam ESHRE/ASRM-sponsored consensus workshop group revised 2003 consensus on diagnostic criteria and long term health risks related to polycystic ovary syndrome (PCOS). Human Reproduction. 2004;19:41-7.

6. Lamarca A, Volpe A. Anti-mullerian hormone $(\mathrm{AMH})$ in female reproduction: is measurement of circulating AMH a useful tool. Clin Endocrinol. 2006;64(6):603-10.

7. Nelson SM, Messow MC, Wallace AM, Fleming R, Mcconnachie A. Nomogram for the decline in serum antimüllerian hormone: a population study of 9,601 infertility patients. Fertil Steril. 2011;95(2):736-41.

8. Van DJ, Faddy MJ, Themmen APN, Jong FH, Peeters PHM, Schouw YT, et al. Relationship of serum antimullerian hormone concentration to age at menopause. J Clinical Endocrinol Metab. 2008;93(6):2129-34.

9. Vet A, Laven JS, Jong FH, Themmen AP, Fauser BC. Antimullerian hormone serum levels: a putative marker for ovarian aging. Fertil Steril. 2002;77(2):357-62.

10. Burger HG, Dudley EC, Hopper JL, Groome N, Guthrie JR, Green A, et al. Prospectively measured levels of serum follicle-stimulating hormone, estradiol, and the dimeric inhibins during the menopausal transition in a population-based cohort of women. J Clinical Endocrinol Metab. 1999;84(11):4025-30.

11. Feyereisen E, Mendez LDH, Taieb J, Hesters L, Frydman R, Fanchin R. Anti-mullerian hormone: clinical insights into a promising biomarker of 
ovarian follicular status. Reprod Biomed Online. 2006;12(6):695-703.

12. Fanchin R, Schonauer LM, Righini C, Guibourdenche J, Frydman R, Taieb J. Serum antimullerian hormone is more strongly related to ovarian follicular status than serum inhibin B, estradiol, FSH and LH on day 3. Hum Reprod. 2003;18(2):323-27.

13. Frattarelli JL, Levi AJ, Miller BT, Segars JH. A prospective assessment of the predictive value of basal antral follicles in in-vitro fertilization cycles. Fertil Steril. 2003;80(2):350-5.

14. Jayaprakasan K, Deb S, Batcha M, Hopkisson J, Johnson I, Campbell B, et al. The cohort of antral follicles measuring 2-6 $\mathrm{mm}$ reflects the quantitative status of ovarian reserve as assessed by serum levels of anti-mullerian hormone and response to controlled ovarian stimulation. Fertil Steril. 2010;94(5):177581.

15. Weenen C, Laven JS, Bergh AR, Cranfield M, Groome NP, Visser JA, et al. Anti-mullerian hormone expression pattern in the human ovary: potential implications for initial and cyclic follicle recruitment. Mol Hum Reprod. 2004;10(2):77-83.
16. Goksedef BP, Idis N, Gorgen H, Asma YR, Api M, Çetin A. The correlation of the antral follicle count and serum anti-mullerian hormone. J TurkishGerman Gynecol Assoc. 2010;11:212-5.

17. Eilertsen TB, Vanky E, Carlsen SM. Anti-mullerian hormone in the diagnosis of polycystic ovary syndrome: can morphologic description be replaced? Hum Reprod. 2012;27(8):2494-502.

18. Rosenfield RL, Wroblewski K, Padmanabhan V, Littlejohn E, Mortensen M, Ehrmann DA. Antimüllerian hormone levels are independently related to ovarian hyperandrogenism and polycystic ovaries. Fertil Steril. 2012;98(1):242-9.

19. Belosi C, Selvaggi L, Apa R, Guido M, Romualdi D, Fulghesu AM, et al. Is the PCOS diagnosis solved by ESHRE/ASRM 2003 consensus or could it include ultrasound examination of the ovarian stroma? Hum Reprod. 2006;21(12):3108-15.

20. Fulghesu AM, Ciampelli M, Belosi C, Apa R, Pavone V, Lanzone A. A new ultrasound criterion for the diagnosis of polycystic ovary syndrome: the ovarian stroma/total area ratio. Fertil Steril. 2001;76(2):326-31.

Cite this article as: Valvekar U, Lakshmi S, Kumar AN. Study of ovarian reserve parameters in infertile women of different age groups. Int J Reprod Contracept Obstet Gynecol 2016;5:2116-21. 